\title{
Prevalence and Antimicrobial Resistance Pattern of Blood Isolates in Patients of Septicemia in ICU: Single Centre Observation
}

ASM Areef Ahsan ${ }^{1 *}$, Kaniz Fatema ${ }^{2 *}$, Lovely Barai ${ }^{3}$, Mohammad Omar Faruq ${ }^{4}$, Fatema Ahmed ${ }^{5}$, Debasish Kumar Saha ${ }^{6}$, Madhurima Saha ${ }^{7}$, Suraiya Nazneen ${ }^{8}$, Tarikul Hamid ${ }^{9}$, Nazia Zabeen ${ }^{10}$

\begin{abstract}
Background: Septicaemia in critically ill patients is a life threatening condition and requires rapid antimicrobial treatment. Infections caused by drug-resistant organisms are more likely to increase risk of death in these patients. The present study was aimed to study the profile of organisms causing septicaemia and their antibiotic resistance pattern in an intensive care unit (ICU) of a teaching hospital in Bangladesh.

Materials and Methods: This cross-sectional study was done in a 21-beded adult ICU of Dhaka city from November 2015 to April 2016.

Results: A total of 362 patients were diagnosed clinically as septicaemia during the study period of six month. 696 blood samples were analyzed and 92 blood samples yielded growth of 94 organisms, which included 89 bacteria and 5 fungal isolates. Mean age of the patients whose blood samples showed growth of organisms was $65.1 \pm 9.1$ years, with female preponderance (56.7\%). High prevalence of diabetes, hypertension and chronic kidney disease was found in these patients. The major organisms isolated were Acinetobacter (29.7\%), Pseudomonas (26.5\%), Klebsiella (18.08\%), Eschericia coli (11.7\%) and Candida (5.3\%). All the isolates were resistant (>50\%) to 3 rd generation cephalosporins. Acinetobacter was highly resistant (>75\%) to most of the antibiotics except colistin. Isolated Pseudomonas was also resistant to aminoglycosides (>90\%) and imipenem (>65\%). Klebsiella was resistant to aminoglycosides and imipenem, but Eschericia coli was sensitive to these antibiotics. Among the Enterobacteriaceae, $81.8 \%$ Eschericia coli and $11.7 \%$ Klebsiella had extended spectrum $\beta$-lactamase activity. Frequency of gram positive organisms (6.38\%) was significantly low in this study $(p<0.05)$.

Conclusion: This study provides information on antibiotic resistance of blood isolates found in ICU patients with septicaemia. It will guide the intensivists to formulate the initial empiric antibiotic therapy for the critically ill patients of ICU.
\end{abstract}

Key Words: Septicaemia, intensive care unit, blood stream infection, bacteria, antimicrobial resistance

\section{Introduction:}

Septicaemia is one of the leading infections in Intensive Care Unit (ICU) patients. The overall case-fatality rate associated with blood stream infection (BSI) is $15 \%$ to $20 \%$, but reaches $35 \%$ to $50 \%$ in ICU patients. ${ }^{1}$ BSI in ICU can either occur secondary to the dissemination of pathogens from a primary focus of infection at a clinical site into the blood stream, or can be primary where the source of infection is unclear. The common foci for secondary BSI in ICU are the respiratory, gastrointestinal and urinary tracts. ${ }^{2}$ Now-a-days, widespread distribution of resistant gram positive and gram negative micro-organisms to currently available antibiotics has gained importance in all over the world. ${ }^{3}$ Septicaemia caused by multi-drug resistant (MDR) organisms is more likely to prolong the hospital stay, increase the risk of death, and require treatment with more expensive antibiotics. Due to wide variations in bacterial drug resistance, results of studies and reports in one region or in a period of time are not necessarily true for other regions or periods of time. ${ }^{4}$

It is therefore essential to document trends in the type of organisms isolated from blood cultures along with their resistance patterns in order to produce meaningful guidelines for the empirical treatment of patients with septicaemia.
Rapid detection and identification of clinically relevant micro-organisms in blood cultures of patients with septicaemia is very essential. Determination of antimicrobial susceptibility pattern for rapid administration of antimicrobial therapy has been shown to reduce the morbidity and mortality associated with BSI. ${ }^{4}$ The objectives of the current study were to describe the frequency of micro-organisms isolated from blood cultures from patients with clinically suspected septicaemia, and the resistance of these bacteria to the commonly available antibiotics.

\section{Materials and Methods:}

This was a cross-sectional study done in the adult ICU of Bangladesh Institute of Research and Rehabilitation in Diabetes, Endocrine and Metabolic Disorder (BIRDEM). This is a tertiary care teaching hospital situated in Dhaka, Bangladesh. The study ICU is a closed ICU dealing with medical, surgical and gynaecological patients. It has 21 beds. Blood for culture was sent from all patients with clinically suspected septicemia who were admitted in this ICU from November 2015 to April 2016.

About $10 \mathrm{ml}$ of blood was collected from two different sites in every patient with strict aseptic precautions. If any patient had central venous line, then one of the two samples were drawn 
Bangladesh Crit Care J September 2016; 4 (2): 100-104

from that line. Samples then immediately put into lytic centrifugation tube, and sent to the Microbiology department of the same hospital. Antimicrobial susceptibility was performed for isolated organisms by Kirby-Bauer's disk diffusion according to Clinical and Laboratory Standards Institute (CLSI) 2015 guidelines. Antibiotic disks were used and the susceptibility patterns of isolated pathogens to commonly used antibiotics were then reported. Extended spectrum beta lactamase (ESBL) testing was performed by combination disc method. All test data were analyzed by the WHONET 5.6 software.

Patients whose blood samples yielded growth of organisms were then identified and their data were collected in a preformed data sheet. Variables like age, gender and presence of co-morbidities were documented.

Statistical analysis has been done by SPSS 17 for windows. Quantitative data has been expressed by percentage, and compared by Z-test of proportion. $\mathrm{P}$ value of $<0.05$ is considered significant.

\section{Results:}

A total of 362 patients were diagnosed as septicaemia during the period of November 2015 to April 2016 in the study ICU and. From these patients, 696 blood samples were analyzed and 92 samples from 67 patients yielded growth of 94 micro-organisms (fig 1). The baseline variables of the patients whose blood culture had growth of micro-organisms is shown in table I.

1. Dr. ASM Areef Ahsan, Associate Professor \& Head, Dept of Critical Care Medicine, BIRDEM General Hospital, Dhaka.

2. Dr. Kaniz Fatema, Assistant Professor, Dept of Critical Care Medicine, BIRDEM General Hospital, Dhaka.

3. Dr. Lovely Barai, Associate Professor \& Head, Dept of Microbiology, BIRDEM General Hospital, Dhaka.

4. Dr. Mohammad Omar Faruq, Professor, Dept of Critical Care Medicine, Ibn Sina Hospital, Dhanmondi, Dhaka.

5. Dr. Fatema Ahmed, Assistant Professor, Dept of Critical Care Medicine, BIRDEM General Hospital, Dhaka.

6. Dr. Debasish Kumar Saha, Registrar, Dept of Critical Care Medicine, BIRDEM General Hospital, Dhaka.

7. Dr. Madhurima Saha, Registrar, Dept of Critical Care Medicine, BIRDEM General Hospital, Dhaka.

8. Dr. Suraiya Nazneen, Registrar, Dept of Critical Care Medicine, BIRDEM General Hospital, Dhaka.

9. Dr. Tarikul Hamid, Medical Officer, Dept of Critical Care Medicine, BIRDEM General Hospital, Dhaka.

10. Dr. Nazia Zabeen, Resident Medical Officer, Dept of Critical Care Medicine, BIRDEM General Hospital, Dhaka.

*First two authors had equal contributions and should be regarded as Principal author

Corresponding Author:

Dr. ASM Areef Ahsan

FCPS (Medicine), MD (Chest), MD (Critical Care Medicine)

Associate Professor \& Head, Dept of Critical Care Medicine

BIRDEM General Hospital

122, Kazi Nazrul Islam Avenue, Dhaka 1000.

E-mail: dr_asmareef@yahoo.com

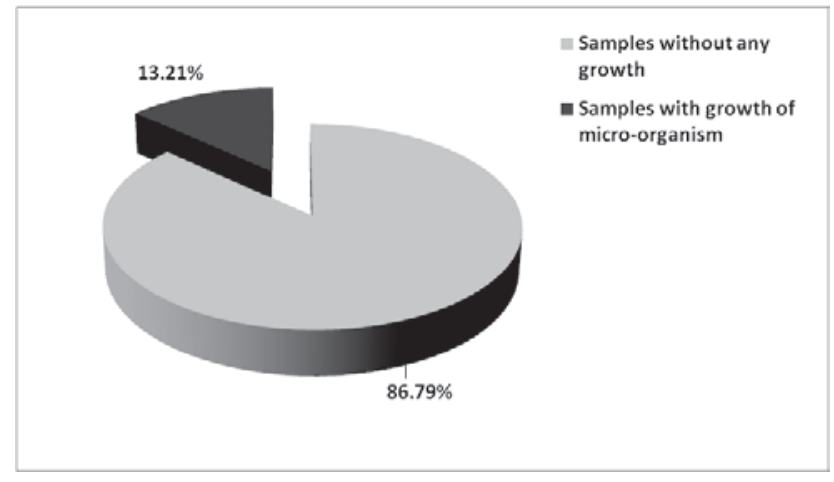

Fig 1: Pie chart showing percentage of sent blood samples which yielded growth of organisms

Table I: Baseline variables of Patients whose blood samples yielded growth of organisms

\begin{tabular}{lll}
\hline $\begin{array}{l}\text { Number } \\
\text { Age (years) }\end{array}$ & 67 & $18.5 \%$ \\
Gender & $65.1 \pm 9.11$ & \\
$\quad$ Male & 29 & $43.2 \%$ \\
$\quad$ Female & 38 & $56.7 \%$ \\
Co-morbidities & & \\
$\quad$ Diabetes Mellitus (DM) & 56 & $83.5 \%$ \\
$\quad$ Hypertension (HTN) & 60 & $89.5 \%$ \\
$\quad$ Chronic Kidney Disease (CKD) & 44 & $65.6 \%$ \\
$\quad$ Bronchial Asthma/Chronic Obstructive & \\
Pulmonary Disease (COPD) & 23 & $34.3 \%$ \\
Cerebro-Vascular Disease (CVD) & 23 & $34.3 \%$ \\
\hline
\end{tabular}

Out of the 94 organisms, 89 were bacterial isolates and 5 were fungal isolates. Figure 2 demonstrated the frequency of isolated organisms from blood cultures. Frequency of gram negative organisms were significantly more than gram positive organisms and fungi. $(88.29 \%$ vs $11.69 \%, p<0.05)$

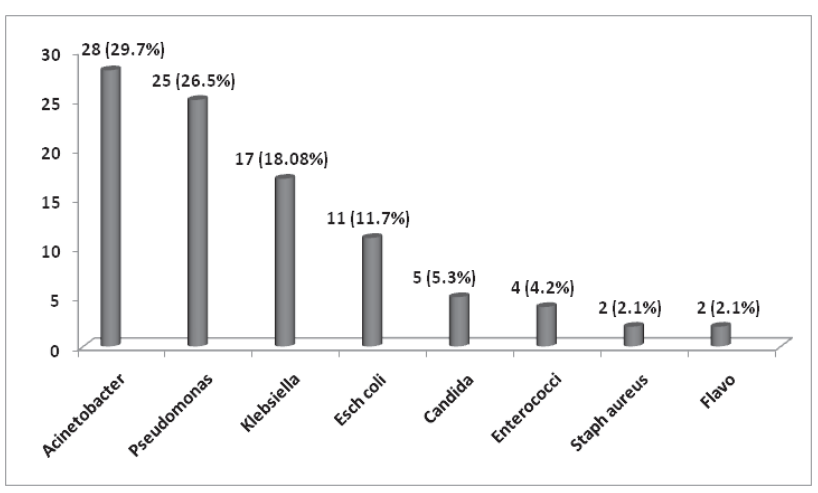

Fig 2: Frequency of isolated organisms from blood cultures

Bacteria abbreviation: Esch coli= Eschericia coli, Flavo= Flavobacteria, Staph aureus $=$ Staphylococcus aureus 
Antibiotic resistance pattern of the common organisms are shown in following figures (fig 3-8). Extended spectrum $\beta$-lactamase (ESBL) activity was found in nine Eschericia coli and two Klebsiella isolates.

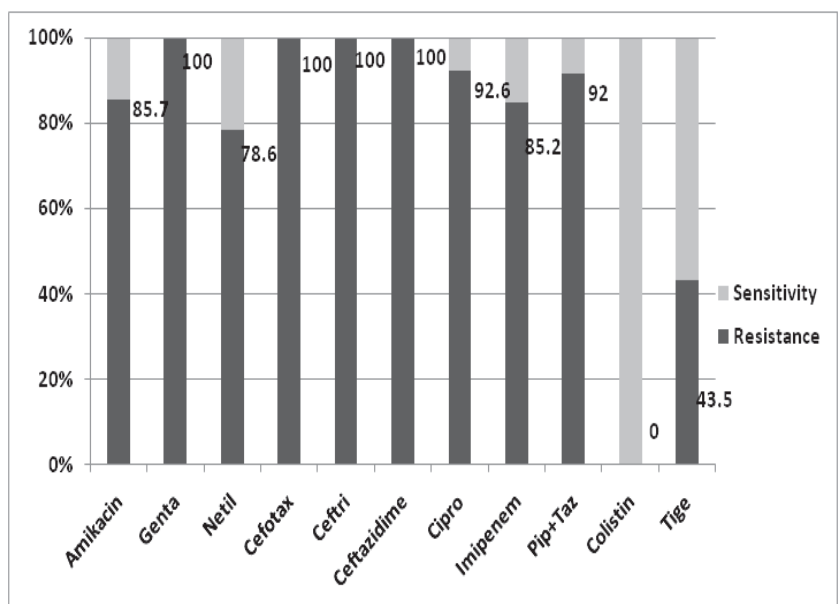

Fig 3: Antibiogram of isolated Acinetobacter* $(n=28)$

*Resistance $\%$ is written beside each bar

Antibiotic abbreviation: Cefotax $=$ cefotaxime, Ceftri $=$ ceftriaxone, Cipro $=$ Ciprofloxacin, Genta $=$ gentamicin, Netil $=$ netilmicin, Pip + Taz $=$ Piperacillin and Tazobactam combination, Tige $=$ tigecycline

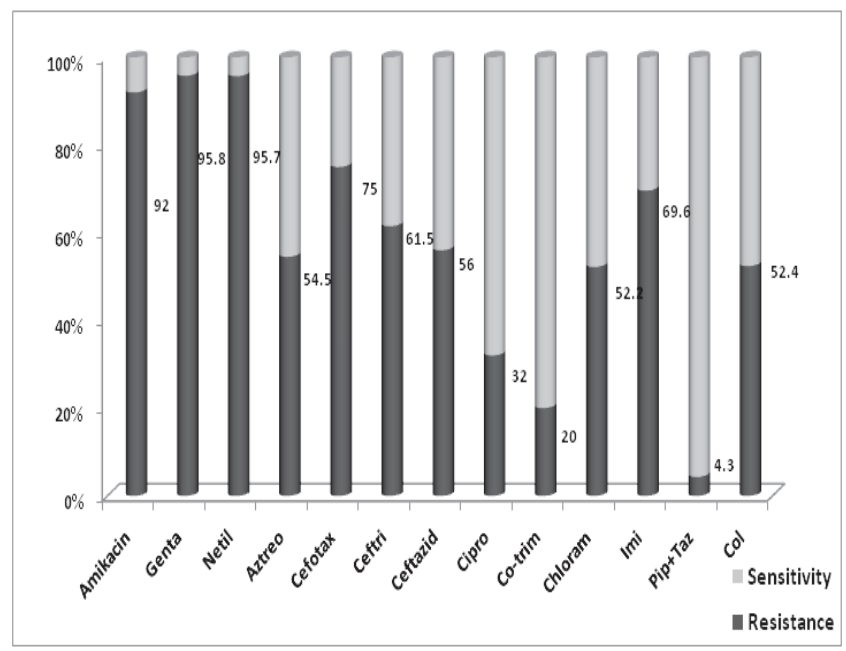

Fig 4: Antibiogram of isolated Pseudomonas* $(n=25)$

*Resistance \% is written beside each bar

Antibiotic abbreviation: Aztreo $=$ aztreonam, Ceftazid $=$ ceftazidime, Cefotax $=$ cefotaxime, Ceftri $=$ ceftriaxone, Chloram $=$ chloramphenicol, $\mathrm{Cipro}=$ Ciprofloxacin, $\mathrm{Col}=$ colistin, Co-trim $=$ co-trimoxazole, Genta $=$ gentamicin, Imi= imipenem, Netil $=$ netilmicin, $\mathrm{Pip}+\mathrm{Taz}=$ Piperacillin and Tazobactam combination

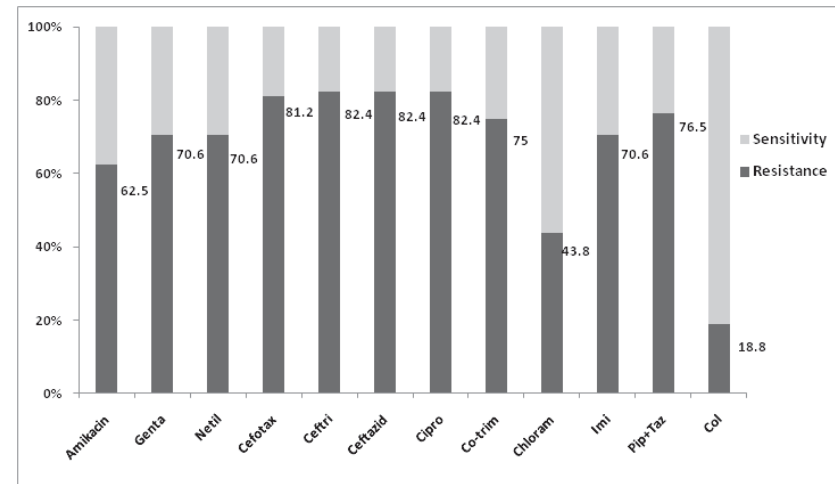

Fig 5: Antibiogram of isolated Klebsiella* $(n=17)$

*Resistance $\%$ is written beside each bar

Antibiotic abbreviation: Ceftazid $=$ ceftazidim, Cefotax $=$ cefotaxime, Ceftri $=$ ceftriaxone, Chloram $=$ chloramphenicol, Cipro $=$ Ciprofloxacin, $\mathrm{Col}=$ colistin, Co-trim $=$ co-trimoxazole, Genta $=$ gentamicin, Imi $=$ imipenem, Netil $=$ netilmicin, Pip + Taz $=$ Piperacillin and Tazobactam combination

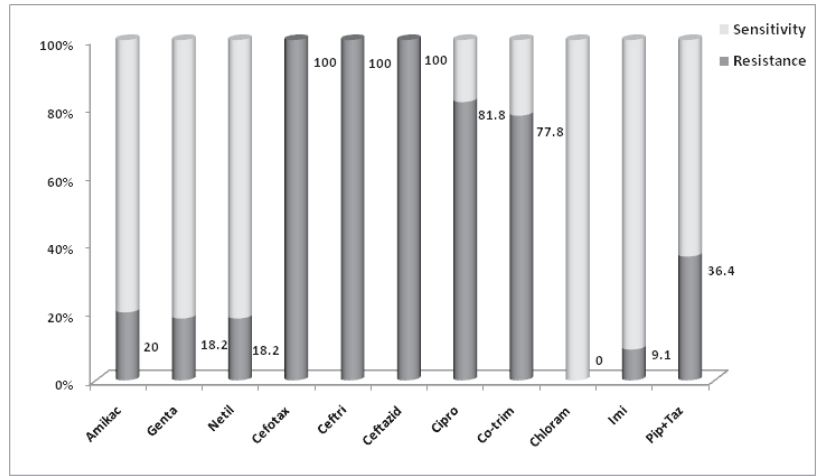

Fig 6: Antibiogram of isolated Eschericia coli $(n=11)$

* Resistance $\%$ is written beside each bar

Antibiotic abbreviation: Amikac $=$ amikacin, Ceftazid $=$ ceftazidim, Cefotax $=$ cefotaxime, Ceftri $=$ ceftriaxone, Chloram $=$ chloramphenicol, Cipro $=$ Ciprofloxacin, Co-trim $=$ co-trimoxazole, Genta $=$ gentamicin, Imi $=$ imipenem, Netil= netilmicin, Pip + Taz $=$ Piperacillin and Tazobactam combination

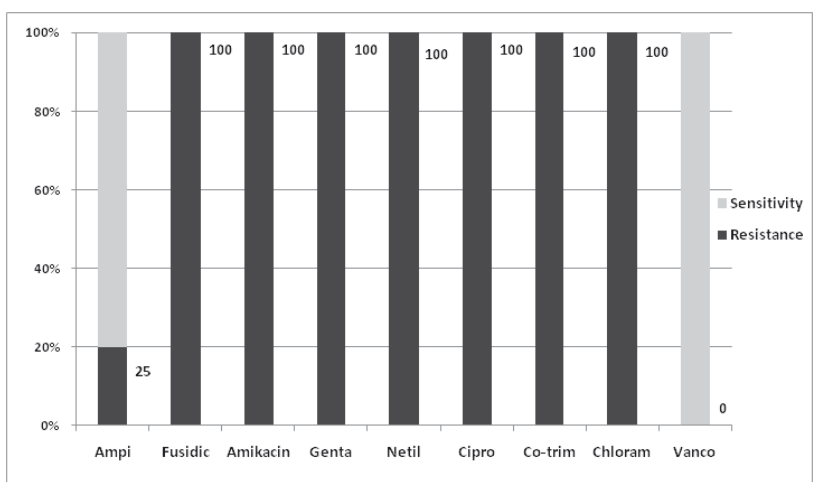

Fig 7: Antibiogram of isolated Enterococci $(n=4)$

* Resistance $\%$ is written beside each bar

Antibiotic abbreviation: Ampi= ampicillin, Chloram= chloramphenicol, Cipro $=$ Ciprofloxacin, Co-trim $=$ co-trimoxazole, Fusidic $=$ fusidic acid, Genta $=$ gentamicin, Netil= netilmicin, Vanco $=$ vancomycin 


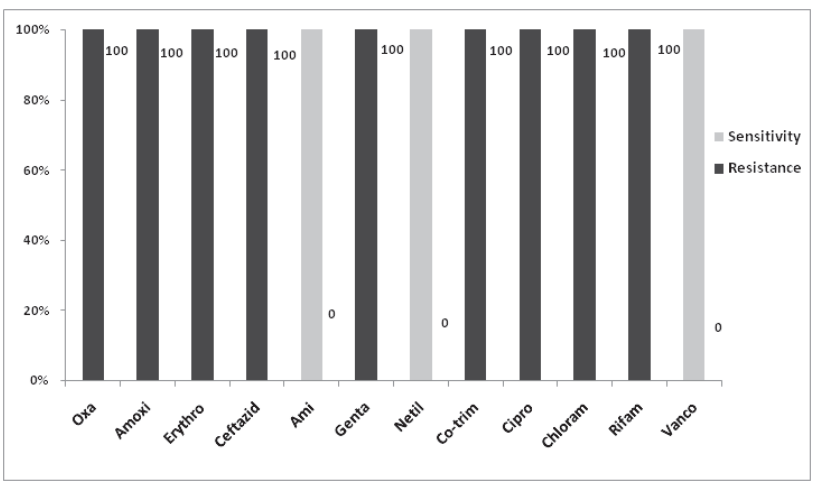

Fig 8: Antibiogram of isolated Staphylococcus Aureus ( $\mathrm{n}=2$ )

* Resistance $\%$ is written beside each bar

Antibiotic abbreviation: $\mathrm{Ami}=$ amikacin, Amoxi $=$ amoxicillin, Ceftazid $=$ ceftazidime, Chloram $=$ chloramphenicol, $\quad$ Cipro $=$ Ciprofloxacin, Co-trim $=$ co-trimoxazole, Erythro $=$ erythromycin, Genta $=$ gentamicin, Netil $=$ netilmicin, Oxa $=$ oxacillin, Rifam $=$ rifampicin, Vanco $=$ vancomicin

\section{Discussion:}

The incidence of septicaemia in Europe and USA has varied from 3.4 to 28 per 1000 hospital admissions..$^{5-7}$ Study of bacteriological profile with antibiotic susceptibility/resistance pattern plays an important role in effective management of bacteraemia cases. Results of our cross sectional study demonstrates the distribution of microbial isolates causing septicaemia in an adult ICU and their resistance pattern to the commonly used antibiotics. Our study revealed that $13.2 \%$ of the samples which were sent from patients with septicaemia were positive for the presence of either bacteria or fungi. This finding is in agreement with study of Culshaw et al (12.2\%). ${ }^{2}$ Though 362 patients were clinically diagnosed as septicaemia, bacteria or fungi were isolated only in $67(18.5 \%)$ patients. As BIRDEM hospital is the largest tertiary hospital dealing with diabetic patients in Bangladesh, so a large number of patients whose blood samples were positive were suffering from DM (83.5\%). Prevalence of other co-morbidities like HTN and CKD was also high, $89.5 \%$ and $65.6 \%$ respectively. But number of patients suffering from bronchial asthma/COPD and CVD was low (34.3\% in both cases).

The spectrum of organisms responsible for bacteraemia has changed. Gram negative bacilli have taken over the gram positive organisms causing septicaemia especially in hospital settings. ${ }^{8,9}$ Mehdinejad et al found $86.5 \%$ gram negative bacteria among all organisms isolated from blood cultures. ${ }^{4}$ Significant number of gram negative organisms were also detected in our study $(88.29 \%$ vs $11.69 \%, p<0.05)$ The severity of the underlying condition of the critically ill patients admitted in ICU and the need for multiple intravascular and other devices make these patients vulnerable to bloodstream infection.

In past, several studies have found Staphylococcus epidermidis to be the most common cause of bacteraemia in the adult ICU, accounting for as many as $57 \%$ of all episodes. ${ }^{10-12}$ This is in contrast to the results of this study, in which Acinetobacter was the commonest isolates found in blood. The isolated Acinetobacter expressed multi-drug resistance pattern. These gram negative cocco-bacilli showed $>75 \%$ resistance to all antibiotics except colistin $(100 \%$ sensitive) and tigecycline (resistance $43.5 \%$ ). Widespread use of carbapenems, especially in ICU settings, is responsible for this high level of resistance. ${ }^{3}$ Easy dissemination of this micro-organism and its ability to acquire multiple resistance mechanisms further complicate the scenario.

Pseudomonas was the $2^{\text {nd }}$ commonest organism isolated from blood of patients with septicaemia in our study. These bacilli showed $>50 \%$ resistance to chloramphenicol, aztreonam, ceftazidime, and ceftriaxone. It was the most common isolates found in blood samples in the same ICU in 2006. ${ }^{14}$ More than $60 \%$ resistance was found against ceftriaxone and imipenem. But resistance of isolated Pseudomonas to aminoglycosides were very high $(>90 \%)$. Sensitivity of this notorious organism to ciprofloxacin, chloramphenicol and combination of piperacillin-tazobactum were good as resistance were less than $35 \%$.

Third commonest organism isolated from blood in the current study was Klebsiella. Resistance of the isolated Klebsiella were $>60 \%$ to aminoglycosides and imipenem, $>75 \%$ to piperacillin and tazobactam combination, and $>80 \%$ to ciprofloxacin and $3^{\text {rd }}$ generation cephalosporins. This carbapenem resistance in this study is much higher than findings by Tabah et al (38\%). ${ }^{15}$ Carbapenems are the $1^{\text {st }}$ line treatment of ESBL producing Enterobacteriaceae. But emergence of carbapenem resistant organisms leave limited therapeutic options and pose a great threat for management of the critically ill patients of ICU.

Eschericia coli were the $4^{\text {th }}$ common isolates in this study. These organisms were highly resistant $(>80 \%)$ to ciprofloxacin and $3^{\text {rd }}$ generation cephalosporins. Resistance was low against piperacillin and tazobactam combination, aminoglycosides and imipenem. Majority (81.8\%) of isolated Eschericia coli were ESBL producing organism. On the contrary, only $11.7 \%$ of isolated Klebsiella were ESBL positive.

Only $6.38 \%$ of isolates found in blood of patients with septicaemia in this study were gram positive organisms. Among them, four were Enterococci and two were Staphylococci. Though none of the isolated Enterococci was resistant to vancomycin, they were $100 \%$ resistant to aminoglycosides, cotrimoxazole and chloramphenicol. On the other hand, both of the isolated Staphylococci were methicillin resistant (100\%). They were also resistant to amoxicillin, ciprofloxacin, cotrimoxazole, chloramphenicol, gentamicin and ceftazidime (100\% to all these antibiotics). Resistance to vancomycin, amikacin and netilmicin was not found in this study. In the study done by Gohel et al, $70.6 \%$ of isolated Staphylococci were methicillin resistant. ${ }^{16}$ In view of significant number of methicillin resistant Staphylococcus aureus (MRSA), drugs like clindamycin, linezolid, and teicoplanin should be considered in the empirical treatment of MRSA.

Candida is the most common nosocomial fungal infection in 
the critically ill patients. Candidaemia in ICU patients is associated with longer hospital stay, higher cost of care and increased morbidity and mortality. Candida spp was the $5^{\text {th }}$ most common isolates in the current study. Reasons for Candidaemia in our patients may be high prevalence of DM, current or previous use of broad spectrum antibiotics, and presence of central venous lines. Unfortunately testing for antifungal drug sensitivity has not been started in our country.

Outcome of these critically ill patients with septicaemia was not analyzed in the present study. Knowledge of epidemiology, risk factors and outcomes of BSIs caused by resistant bacteria has a major influence on global management of ICU patients. So, further study may be done to evaluate all these factors in ICU patients with septicaemia.

\section{Conclusion:}

Findings of the present study showed that gram negative organisms dominated the blood isolates in patients with septicaemia with Acinetobacter in leading position. All gram negative organisms were highly resistant $(>60 \%)$ to $3^{\text {rd }}$ generation cephalosporins. Acinetobacter, Pseudomonas and Klebsiella were resistant $(>60 \%)$ to aminoglycosides and imipenem. Majority of isolated Eschericia coli were ESBL-positive. Though there was no vancomycin-resistant Enterococci (VRE), all isolated Staphylococci were methicillin-resistant. The rise in antibiotic resistance in blood isolates emphasizes the importance of hospital infection control policies, rational antimicrobial prescribing practices, and invention of new antimicrobial drugs and vaccines. Our results will provide a useful guideline for selecting empirical antibiotics in critically ill patients with septicaemia.

\section{References:}

1. Russotto V, Cortegiani A, Graziano G et al. Bloodstream infections in intensive care unit patients: distribution and antibiotic resistance of bacteria. Infection and Drug resistance 2015; 8: 287-96.

2. Culshaw N, Glover G, Whiteley $\mathrm{C}$ et al. Healthcare-associated bloodstream infections in critically ill patients: descriptive cross-sectional database study concordance with clinical sites isolates. Annals of Intensive Care 2014; 4:34.

3. Cortes JA, Leal AL, Montanez AM et al. Frequency of microorganisms isolated in patients with bacteremia in intensive care units in Colombia and their resistance profiles. Braz J Infect Dis $2013 ; 17$ (3): 346-52.
4. Mehdinejad M, Khosravi AD, Morvaridi A. Study of prevalence and antimicrobial susceptibility pattern of bacteria isolated from blood cultures. Journal of Biological Sciences 2009; 9(3):249-53.

5. Williams GM, Houang ET, Shaw EJ et al. Bacteraemia in a London teaching hospital 1966-75. Lancet 1976; 2:1291-3.

6. Weinstein MP, Reller LB, Murphy JR et al. The clinical significance of positive blood cultures: a comprehensive analysis of 500 episodes of bacteraemia and fungaemia in adults, laboratory and epidemiologic observations. Rev Infectious Disease 1983; $5: 35-53$.

7. McGowan JE, Barnes MW, Finland M. Bacteraemia at Boston city hospital: occurrence and mortality during 12 selected years (1935-1972) with special reference to hospital acquired cases. J Infect Dis 1975; 132: 316-34.

8. Kang CI, Kim SH, Park KD et al. Bloodstream infections caused by antibiotic-resistant gram-negative bacilli: risk factors for mortality and impact of inappropriate initial antimicrobial therapy on outcome. Antimicrob Agents Chemother 2005; 49:760-66.

9. Kumar S, Rizvi M, Vidhani S, Sharma VK. Changing face of septicaemia and increasing drug resistance in blood isolates. Indian J PatholMicrobiol 2004; 47: 441-46.

10. Spencer RC. Epidemiology of infection in ICUs. Intensive Care Medicine 1994; 20(supplement4):2-6.

11. Pittet D, Tarara D, Wenzel RP. Nosocomial bloodstream infection in critically ill patients.JAMA 1994; 271:1598-1601.

12. Rello J, Ricart M, Mirelis B et al. Nosocomial bacteraemia in a medical-surgical intensive care unit: epidemiologic characteristics and factors influencing mortality in 111 episodes. Intensive Care Medicine 1994; 20:94-98.

13. Patwardhan RB, Dhakephalkar PK, Niphadkar KB, Chopade BA. A study on nosocomial pathogens in ICU with special reference to multiresistant Acinetobacter baumanii harbouring multiple plasmids. Indian J Med Res 2008; 128:178-87.

14. Barai L, Fatema K, Haq JA et al. Bacterial profile and their antimicrobial rsistance pattern in an intensive care unit of a tertiary care hospital in Dhaka. Ibrahim Med Coll J 2010; 4(2): 66-9.

15. Tabah A, Koulenti D, Laupland K et al. Characteristics and determinants of outcome of hospital-acquired bloodstream infections in intensive care units: the EUROBACT International Cohort Study. Intensive Care Med 2012; 38 (12): 1930-45.

16. Gohel K, Jojera A Soni S et al. Bacteriological profile and drug resistance patterns of blood culture isolates in a tertiary care nephrourology teaching institute. BioMed Research International 2014 ; 